\title{
Configurações
}

Revista Ciências Sociais

$14 \mid 2014$

Para além da Governação. Políticas, práticas e discursos de inclusão e promoção da diversidade cultural

\section{El problema de la deserción escolar y las posibilidades de la investigación-acción participativa}

O problema do abandono escolar e as possibilidades da investigação-ação participativa

Participatory Action Research for school retention of adolescent girls at risk of dropping out in Saltillo

\section{Gabriela de La Peña Astorga}

\section{(2) OpenEdition}

\section{Journals}

Edición electrónica

URL: http://journals.openedition.org/configuracoes/2309

DOI: $10.4000 /$ configuracoes.2309

ISSN: $2182-7419$

Editor

Centro de Investigação em Ciências Sociais

\section{Edición impresa}

Paginación: 129-149

ISBN: 1646-5075

ISSN: 1646-5075

\section{Referencia electrónica}

Gabriela de La Peña Astorga, «El problema de la deserción escolar y las posibilidades de la investigación-acción participativa », Configurações [En línea], 14 | 2014, Publicado el 30 marzo 2015, consultado el 10 diciembre 2020. URL : http://journals.openedition.org/configuracoes/2309 ; DOI : https://doi.org/10.4000/configuracoes.2309 


\title{
El problema de la deserción escolar y las posibilidades de la investigación-acción participativa
}

\author{
o problema do abandono escolar e as possibilidades da investigação-ação \\ participativa \\ Participatory Action Research for school retention of adolescent girls at risk of \\ dropping out in Saltillo
}

\section{Gabriela de La Peña Astorga}

1 El problema de la deserción escolar en México es tal que, a nivel nacional, un $43 \%$ de la población entre 15 y 19 años, no asiste a la escuela. Este porcentaje representa a 4 millones 741 mil jóvenes fuera del sistema educativo, de acuerdo con el II Censo de Población y Vivienda del año 2010 (INEGI, 2011). Por su parte, una primera aproximación a las razones de deserción en este segmento de edad, se llevó a cabo a partir del Censo General de Población y Vivienda del 2000. Al analizar dichos datos, Navarro (2001) concluyó que de los jóvenes que desertaron del sistema educativo, 37\% lo hizo porque no quiso o no le gustó estudiar, $35 \%$ lo dejó por problemas económicos; $6 \%$ porque se casó o unió; $5 \%$ por considerar que había terminado sus estudios, y un $2 \%$, declaró que fue porque estaba lejos la escuela o no había (pp. 48 y 49).

2 Los porcentajes anteriores son equiparables a los que se presentan en distintos países latinoamericanos y en el norte del Continente, de acuerdo con el análisis presentado en el Reporte de la Encuesta Nacional de Deserción de Educación Media Superior (SEP y COPEEMS, 2012). Este cúmulo de datos generados en los niveles nacional, internacional, regional y local, ha permitido clasificar las causas de deserción en exógenas y endógenas, o personales e institucionales, entre otras categorías. No obstante que estas tipificaciones permiten aclarar el panorama de los factores predictivos de la deserción escolar y profundizar en el estudio o intervención de las variables predominantes en grupos juveniles específicos, se acepta de forma generalizada entre los estudiosos y los gestores 
educativos que el problema de la deserción escolar de los 15 a los 19 años, responde a una realidad compleja y multifactorial que requiere abordajes y estrategias de intervención adecuadas a esta naturaleza del fenómeno.

3 Para el caso de nuestro país, encuestas posteriores al Censo de Población y Vivienda del 2000, han mostrado una radiografía más detallada que incluye el papel que juegan factores como la edad, el género, la composición familiar o la ubicación geográfica. Así, encontramos que aunque los motivos personal y económico siguieron constituyendo más del 70\% de las respuestas de los jóvenes en la encuesta aplicada por el IMJUVE en el año 2005; para el año 2009 la Encuesta Nacional de Ocupación y Empleo (ENOE-INEGI, 2010) reportó que el 52\% de las razones de deserción escolar en secundaria era la sumatoria de las opciones "insuficiencia de dinero para pagar la escuela" y "necesidad de aportar dinero al hogar", así como que el tercer lugar lo ocupaba la respuesta "embarazo, matrimonio y unión" con un 12\%; y, contrario a los datos del año 2000, en cuarto lugar estaba "no le gustó estudiar" con un $11 \%$ de respuesta.

Así mismo, llama la atención en los resultados arrojados por la ENOE (INEGI, 2010) que mientras tanto para hombres como para mujeres la primera razón de deserción es económica, la segunda difiere de acuerdo con el género: en el caso de las mujeres, "embarazo, matrimonio o unión" se presenta en segunda posición con un 23\%; para los hombres, se ubica con un $27 \%$ de respuesta la opción "necesidad de aportar dinero al hogar" en segundo lugar. También destaca en esta encuesta que los factores escolares ocupan tan sólo un $2,5 \%$ de las razones de deserción con la opción "reprobación, suspensión o expulsión". Finalmente, una encuesta más reporta para el año 2010 la respuesta que dieron los directores de las preparatorias del país en las que se aplicó la prueba ENLACE sobre lo que estos consideraban las razones de deserción en sus escuelas: $43 \%$ respondió que fue por problemas económicos, $24 \%$ que fue por falta de interés de los estudiantes, y el 19\%, que fue por bajo rendimiento (SEP y COPEEMS, 2012).

Coahuila se ubica, a nivel nacional, como la segunda entidad federativa con mayor índice de deserción de secundaria, con un índice del 7.9 y sólo después del Estado de Michoacán (SEP, 2012), a pesar de que su promedio de escolaridad es mayor al del país: si para la República Mexicana es de 8.6 grados (equivalente a un poco más de segundo grado de secundaria), en Coahuila alcanza el 9.5 (poco más de tercero de secundaria) según datos del II Censo de Población y Vivienda (INEGI, 2010). Si a lo anterior agregamos que nuestro estado contaba hasta el año 2012 (INEGI, 2013) con el mayor índice de embarazos adolescentes del país, con un $23,1 \%$ del porcentaje de nacimiento de hijos de madres menores de 20 años ${ }^{1}$, el problema de la deserción se recrudece aún más para el segmento poblacional de los 15 a los 19 años en el Estado de Coahuila. En esta zona del país, la situación se complejiza para las mujeres adolescentes, no sólo por los obstáculos arriba mencionados, si no por verse inmersas en un contexto cultural que valora poco su preparación académica al dar prioridad a su desempeño en otros ámbitos como el laboral o el de la asistencia familiar².

6 Conscientes de esta problemática, diversas instituciones e iniciativas sociales intentan apoyar la permanencia de las adolescentes en situación de riesgo de deserción escolar a través de programas o proyectos que estimulen la valoración de su preparación académica en pro de hacerlas constructoras de comunidades más equitativas. Así, tanto desde el sector público (DIF, SEP) como desde el privado (Organismos de la Sociedad Civil -OSC-, fundaciones diversas), se realizan tareas para detectar estos casos de riesgo y coaduyar a su permanencia en la educación formal de la secundaria y la preparatoria. 
7 En Saltillo, diversas instituciones de benefi cencia pública ${ }^{3}$ mantienen programas activos para niños y adolescentes en situación de riesgo de deserción; pero hasta ahora, no han logrado vincular sus esfuerzos con lo que podrían aportar los conocimientos, habilidades y valores del campo de la comunicación; sobre todo a través de un trabajo colaborativo que vincule comunidades enmarcadas en la diversidad cultural y con diferentes posibilidades de intervención social, tales como dichas OSCs, programas del sector público así como estudiantes y profesores de distintos niveles educativos. En este contexto, es fundamental desarrollar proyectos de intervención y de investigación que al tiempo en que fomentan una cultura de desarrollo equitativo, permiten aproximarse con todas las herramientas científicas a la comprensión y propuesta de solución de un problema tan relevante para nuestro país como lo es la deserción escolar adolescente. Con lo anterior nos referimos a la propuesta de un trabajo transdisciplinar en la que diversos agentes sociales se avoquen a la búsqueda de soluciones a través de un análisis, una toma de decisiones y una ejecución de acciones colaborativa e inclusiva ${ }^{4}$. Esta forma de trabajo es la que, desde un marco de acción democrático, ha sido resaltada desde el campo de la comunicación para el desarrollo como idónea para alcanzar los objetivos de investigación-acción en atención a problemáticas específicas 5 .

8 Aunque desde otros campos sociales y humanísticos se ha abordado la problemática de la deserción y ésta aparece de manera reiterada dentro de los planes de desarrollo a nivel federal, estatal y municipal; desde el campo de la comunicación ha sido poco frecuentada como problema de investigación-acción que enlace el origen socio-económico, institucional y cultural del abandono escolar con las herramientas metodológicas y profesionales de una comunicación compleja y socialmente vinculante. Es el boliviano Luis Ramiro Beltrán (2005), principalmente, quien se da a la tarea -siguiendo a Paulo Freire (1973, 2006, 2007, 2009), a Javier Kaplún (1987) y Mario Kaplún (1997), entre otros investigadores de la educomunicación- de ejecutar proyectos de empoderamiento comunitario en América Latina que no soslayen ni las condiciones periféricas de los grupos a intervenir ni el repertorio cultural con el que estos cuentan y que puede apuntalarse como la vía participativa para el mejoramiento de su calidad de vida.

\section{La construcción del modelo de IAP}

El modelo que se presenta a continuación, fue realizado a partir de la experiencia de intervención que inició en el año 2010 con la impartición de una serie de talleres vinculantes sobre competencias de escritura creativa en el Centro de Superación "Estela V. Barragán", A.C. (CSEVB), escenario de este estudio ${ }^{6}$, y continuó su propio curso de desarrollo durante los tres años siguientes, como se explicará más adelante. De este modo, lo que aquí se sintetiza como modelo de intervención-investigación, ha sido construido participativamente con los involucrados en esta relación de intercambio, que ha integrado desde entonces a 20 estudiantes de la Facultad de Ciencias de la Comunicación (FCC) de la Universidad Autónoma de Coahuila (UAdeC) en 5 generaciones distintas, 5 estudiantes de otras universidades y preparatorias de la Ciudad (ITESM, UVM), 3 tesistas de la FCC y 3 especialistas en temas de desarrollo humano (una de ellas, catedrática de la FCC); por parte de la OSC con la cual se estableció la vinculación, han formado parte del proyecto 15 mujeres adolescentes en situación de riesgo de deserción y sus familias así como 5 mujeres de su consejo directivo. 
10 En el año 2012, el proyecto fue presentado al Programa de Mejoramiento del Profesorado (PROMEP) como propuesta de investigación-acción y fue aprobado con la hipótesis, los objetivos y metodología siguientes, lo que permitió sistematizar la experiencia y re direccionar la investigación hacia las categorías que habían emergido como prioritarias para entender/atender el problema de la deserción adolescente en Saltillo desde los campos de la comunicación para el desarrollo y la educomunicación.

\section{Hipótesis, objetivo general, escenario de investigación-intervención y metodología}

11 El campo de la comunicación, con sus múltiples vertientes, se constituye como una arena ideal para apoyar el desarrollo sostenido de las comunidades, dentro de las cuales, el estado de la educación -formal e informal- juega un papel decisivo. Los contenidos (en conocimientos, habilidades y valores) de las áreas de aplicación de la comunicación (expresión verbal y escrita, comunicación interpersonal, intercultural, intergeneracional, grupal, masiva o la comunicación a través de nuevas tecnologías, entre otros) pueden fomentar que mujeres adolescentes en situación de riesgo de deserción escolar logren establecer estrategias para permanecer en el sistema de educación formal (secundaria y preparatoria) y contribuyan al cambio en sus comunidades para abordar y trabajar las causas que hacen que esta problemática endémica pueda revirar hacia la generación de un entorno más favorable para su desarrollo académico y social.

Por otra parte, las herramientas metodológicas de una perspectiva de investigaciónacción aplicada a la problemática de la permanencia escolar en mujeres adolescentes que se encuentran en situación de riesgo, permiten alcanzar dos objetivos generales:

13 1. Contribuir al empoderamiento de los sujetos de intervención, al propiciar procesos democráticos, participativos y de autogestión del desarrollo comunitario e individual desde las herramientas, conocimientos y valores que puede aportar el campo académico de la comunicación e,

14 2. Investigar a profundidad las variables que se ven implicadas en la problemática de estudio, desde un abordaje transdisciplinar que ubique el ángulo de estudio en la complejidad socio-cultural y económica en que se dan los fenómenos de comunicación comunitaria; específicamente, bajo el enfoque de la comunicación para el desarrollo.

Como se ha mencionado anteriormente, el escenario de investigaciónacción fue el Centro de Superación "Estela V. Barragán", A.C, de Saltillo. Registrada como OSC en el padrón del DIF-Coahuila así como reconocida en sus planes de estudio por la SRE, el CONEVYT y el INEA a partir del año 2000, la organización fue fundada por la Profra. Estela V. Barragán ${ }^{7}$ en 1973, cuando ésta inició un club de lectura para mujeres en su casa. La problemática que atendía fundacionalmente estaba relacionada con las brechas de conocimiento intergeneracionales y los valores familiares desde una perspectiva de género. Desde entonces, la A.C. busca impactar socialmente a través del papel formativo de la mujer en la familia, y actualmente cuenta con un promedio anual de 150 alumnas y asociadas activas.

En 1994, da inicio a su "Programa Ayuda a la Esperanza" (PAE), cuyo objetivo es "brindar a sus beneficiarias la esperanza de una vida mejor a través de la educación", dirigido a hasta 20 niñas (de primaria, secundaria, preparatoria y, desde agosto del 2013, se cuenta con las dos primeras beneficiarias que accedieron a estudios universitarios) de zonas 
marginales de la Ciudad, que cuenten con las siguientes características: a) liderazgo en sus escuelas, actitud de superación a través de la educación, b) situación de riesgo de deserción debido a condiciones sociales, económicas o institucionales, y c) que cuenten con acompañamiento familiar. El PAE es una iniciativa financiada por la Asociación, que ofrece los siguientes beneficios a las niñas que participan en él y que fueron seleccionadas tanto por el consejo directivo de la A.C. como por las directoras o directores de sus escuelas: a) beca mensual de 200 pesos, que se entrega a las madres o tutoras para requerimientos escolares, b) clases de reforzamiento escolar y habilidades para el cambio individual (superación) y social, c) tutoría por parte de miembros de la A.C. y de otras instituciones, d) programa de nutrición y de atención a las madres de las niñas y adolescentes, que acuden juntas todos los sábados, en un horario de 8:00 a 13:00 horas, al recinto de la Asociación.

De acuerdo con los objetivos planteados para este proyecto, el enfoque que se utilizó en la investigación fue mayormente cualitativo, basado en las propuestas de la Teoría Fundamentada de Strauss y Corbin (2002) así como en las del interaccionismo simbólico de George H. Mead y Herbert Blumer (1969). Lo anterior implicó distintos momentos de investigación que requirieron la utilización de técnicas como el análisis documental, la entrevista en profundidad, la discusión de grupos, la observación participante y la construcción de historias de vida escolares y familiares ${ }^{8}$. Estos resultados, permitieron ir perfilando la estrategia y los contenidos del proyecto de intervención así como ir ajustando el modelo que a continuación se presenta.

\section{El modelo de IAP y sus fases de aplicación}

Diversos autores definen a la investigación-acción participativa (IAP) como el enfoque de aproximación a una problemática social donde se busca la transformación del grupo que participa en él, para orientarse hacia el incremento de su calidad de vida a través de acciones educativas, de investigación y de participación equitativa (Alcocer, 1998; Álvarez-Gayou, 2010; Hernández Sampieri, Fernández Collado y Baptista Lucio, 2010). Lo anterior implica procesos paralelos (investigación e intervención que surgen simultáneamente de la acción participativa del grupo conformado por actores sociales que viven e investigación la problemática), que pueden entrecruzarse en los momentos de IAP que sea necesario para alcanzar un objetivo general busca conocer desde una perspectiva subjetiva la problemática que caracteriza al (o enfrenta el) grupo -a través de actividades que fomenten el autoconocimiento y la revelación consciente de la problemática- así como proponer una estrategia de solución formulada desde la discusión de experiencias y conocimientos de los miembros del grupo en una relación dialógica para a avanzar paulatinamente en la autogestión para ese y otros problemas que se detecten a corto, mediano y largo plazo.

Si bien es cierto que algunos autores se refieren a la investigación-acción como vía para "resolver problemas cotidianos e inmediatos" (Álvarez-Gayou, 2010), aquí diferimos de la percepción de que este método de trabajo prácticocientífico tenga límites espaciales o temporales particulares y sostenemos que es precisamente la profundización cada vez mayor en el conocimiento e intervención de las problemáticas sociales que van siendo detectadas por el grupo que interactúa a través la IAP, lo que hace de este enfoque de investigación una herramienta transformadora ${ }^{9}$ que, como apuntan León y Montero (cit. 
En Hernández Sampieri et al., 2010), puede crecer en “espirales” cada vez más especializadas e incluyentes ${ }^{10}$.

Murcia Peña y Jaramillo Echeverry (2000) resumen las etapas de la IAP en tres momentos:

En el primero se exige un alto contenido de práctica mediante el intercambio de relaciones comunitarias; en el segundo momento se genera un proceso de reflexión en la identificación y priorización de problemas; y por último se llega a una práctica enriquecida que en su proceso se autotransforma por medio de la puesta en marcha del proyecto comunitario por parte de todos los sujetos de la comunidad. (p. 75)

21 Lo anterior ha sido adaptado al trabajo que fundamenta el modelo que proponemos a continuación (ver Fig. 1), donde la inmersión comunitaria (realizada en el año 2010) permitió detectar como eje de IAP el problema de la deserción escolar en mujeres adolescentes así como a la comunicación familiar como uno de los principales factores de protección para la permanencia escolar, que habrían de ser abordadas bajo los siguientes principios éticos y operativos:

22 1. Tipos de observación / avances de investigación: a) no intrusiva / exploratoria, b) abierta: se da a conocer el papel del investigador(a), c) participante, d) consciente del tipo de relación que se establece, e) negociada en aplicación y alcances, f) basada en el intercambio, g) de post-indagación, acuerdos para el seguimiento y la evolución de la vinculación.

23 2. Acuerdos sobre el equipamiento y su uso para la investigación-intervención: a) libreta, b) uso de cámaras fotográfica y de video, c) uso de grabadora de audio, d) utilización de programas informáticos, e) patrocinios para material y consumibles.

24 3. Responsabilidad de la vinculación tanto con el grupo de estudio como con otros entes individuales o institucionales involucrados en el proyecto: a) protección de informantes o comunidad de investigación-acción, b) compartir resultados con la comunidad de IAP, tanto cotidianamente como a través de documentos generados a lo largo de la investigación, c) presentar opciones o propuestas, d) trabajar objetivos de desarrollo de forma participativa, e) negociar permisos y acuerdos para publicaciones sobre los resultados.

25 4. El proceso de investigación y de trabajo en proyectos de desarrollo debe incluir en primer término los siguientes valores: dignidad de la persona (protección de su identidad y su autoestima), crecimiento en conocimientos y habilidades para todos los involucrados. Si la investigación la realiza un equipo de trabajo, es necesario que todos los involucrados en ella tengan como prioridad estos valores.

26 5. Confidencialidad: manejo de la misma a lo largo de todas las etapas de la investigación, en pro de la comunidad de estudio y de los resultados de la investigación.

27 6. Transparencia: tanto el proyecto de desarrollo para el grupo de estudio como la investigación, deben tener claramente establecidos los objetivos, de modo que desde un primer momento todos los involucrados sepan en qué etapa se encuentran, qué metas persiguen y cuál sería la mejor estrategia para lograrlo.

28 7. Apego a la verdad científica. El objetivo de toda investigación, más allá del proyecto de desarrollo que la acompaña y sostiene, es obtener información confiable, veraz y que aporte al conocimiento de la problemática. Por lo tanto, es posible que algunas entidades públicas o privadas se consideren "mal retratadas" a través de los resultados. En ningún momento el objetivo de la investigación debe ser señalarlas con el dedo (esto va en contra de los principios de las ciencias sociales o humanas), pero habrá que considerar como 
parte de la etapa de publicación de resultados que esto podría presentarse y dejar en claro que la ciencia, por definición, busca el conocimiento profundo de las problemáticas para poder llegar a soluciones que permitan el progreso social y comunitario.

8. Los medios de comunicación. Estar atentos todos los involucrados a que no se tergiverse la información o asegurarnos de explicar todo lo que sea necesario. Buscar que no se pierda el espíritu que inspiró todo el proyecto, a saber: buscar el crecimiento tanto de la comunidad de estudio como del proceso de conocimiento que implica hacer investigación-acción. Los medios de comunicación (tradicionales o a través de las nuevas tecnologías), pueden ser aliados en la consecución de las metas de desarrollo social. Fig. 1. Modelo de IAP. Comunicación para la permanencia escolar de mujeres adolescentes en situación de riesgo de deserción.

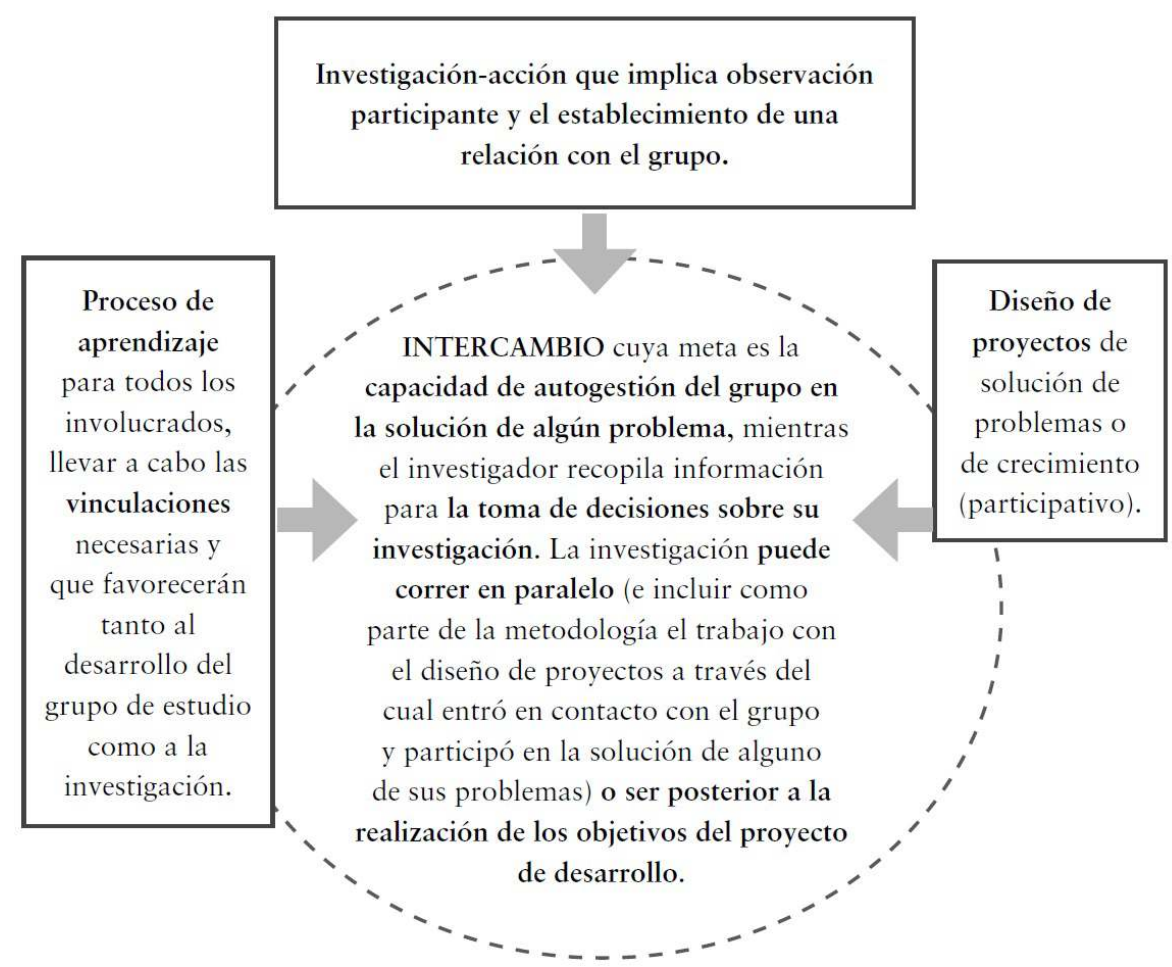

Por su parte, el abordaje del proyecto ha sido desarrollado teniendo como problema de intervención propiciar el desarrollo de competencias, valores y conocimientos de la comunicación para el cambio social (valoración de la educación en la familia) en adolescentes en situación de riesgo de deserción así como ha corrido en paralelo con la pregunta de investigación ¿de qué forma influye la comunicación/interacción familiar en la permanencia escolar de la comunidad de estudio?

Las fases que han dado lugar a las actividades de IAP, se presentan en el siguiente cuadro (ver Tabla 1) y se explican a continuación:

Las fases 1 y 2 iniciaron en marzo de 2009 con el primer grupo de IAP formado por 5 estudiantes de la FCC que habían tomado un curso de "Historias de vida" como taller extracurricular conmigo. Como parte del seguimiento al taller, habríamos de elegir un escenario de investigación y partir desde la problemática que emergiera como propia del grupo de estudio para construir con ellos sus biografías. Yo había trabajado con anterioridad (2008-2009) en el CSEVB, con otros grupos de estudiantes que cursaban una materia con enfoque de aprendizaje-servicio, así es que la entrada al escenario estaba 
hasta cierto punto garantizada. Nos enfocamos en las adolescentes participantes en el PAE, con quienes establecimos una relación de intercambio y aproximación ofreciendo un curso de escritura creativa para ellas junta con la instructora voluntaria del CSEVB con quien ya tomaban clases de literatura. Durante esta primera experiencia de acercamiento, decidimos -entre los 7- los objetivos de aprendizaje e investigación, que discutimos posteriormente con ellas. Acordamos que el objetivo del taller fuera la escritura asesorada por los estudiantes de la FCC en pares de trabajo: una adolescente, un estudiante- de un cuento con temática de salud.

Como la educación sexual aparecía en la literatura de los estudios de juventud como prioridad a atender, decidimos proponerles que el ejercicio de escritura fuera el siguiente: construir un cuento con el detonador "un personaje (hombre o mujer) se encuentra en un hospital donde lo acaban de diagnosticar con VIH". A partir de ese arranque, recorrimos en el curso algunas aproximaciones a una estructura básica de narrativa (cuento, específicamente) y les pedimos que tanto la historia como los personajes estuvieran fundamentados en investigación documental o de campo. Sobre la marcha de la construcción de las historias, el equipo inicial de IAP decidimos organizar un evento público en el CSEVB donde ellas pudieran ejercitar sus competencias de expresión oral y vivir la experiencia de compartir con una comunidad sus productos creativos. En febrero de 2010, se llevó a cabo la presentación del proyecto "Érase una vez..." (título elegido por las participantes para el evento) ante las personas que acudían los sábados al PAE (sus madres, tutoras, asesoras y directivas del CSEVB), donde las adolescentes tomaron el micrófono y leyeron una parte de sus cuentos. También repartimos como libro de bolsillo la impresión de sus productos, que había sido diseñado gráficamente por un estudiante miembro del equipo de IAP. En esta primera experiencia se establecieron las reglas éticas y operativas del proyecto, así como una primera versión del modelo que se describe al inicio de este subtema. Al final de la presentación, se abrió un foro de discusión entre los presentes que dio origen a la siguiente fase del proyecto, entregamos diplomas de participación y tuvimos una sesión fotográfica. Como resultado obtuvimos una invitación a seguir trabajando en el CSEVB con otros talleres para las adolescentes, la solicitud de integrar a las madres de familia en el trabajo que se hacía con ellas, así como una primera sesión de retroalimentación bajo un espíritu de equipo cohesionado entre los integrantes de la IAP y las adolescentes del CSEVB. Abrimos una página en FB para mantenernos en contacto y compartir fotos, que sigue funcionando a la fecha; y como resultado de la investigación pudimos contar con un panorama de los factores predictivos de deserción escolar en el grupo (desestructuración familiar, necesidades económicas, y embarazo temprano; para el caso de las adolescentes en el CSEVB, en esa secuencia de aparición) que nos permitiría explorar posibilidades de intervención desde el campo de la comunicación.

Por otra parte, pudimos establecer una dinámica de trabajo para cada sesión del taller que también era nuestro momento de trabajo de campo-, que es la siguiente y que hemos conservado hasta la fecha cuando se integran nuevos miembros al equipo de IAP: nos reuníamos previamente en la FCC para diseñar la sesión y discutir observaciones y bibliografía; teníamos una junta de 20 minutos antes de entrar los sábados al escenario de estudio en un jardín público para acordar responsabilidades y la dinámica de la sesión; nos reuníamos en el mismo jardín al terminar la sesión para intercambiar notas de campo, observaciones y responsabilidades para el seguimiento del proyecto y del llenado de los instrumentos de la investigación. También, abrimos un grupo en Facebook para 
intercambiar documentos en línea entre los miembros del equipo de IAP, que continúa activo a la fecha. El trabajo de los estudiantes de la FCC en estas dos primeras fases, fue voluntario y no implicó Servicio Social (SS), investigación de tesis ni créditos cocurriculares para ellos; pero sí fue posible vincularnos con otros estudiantes que hacían SS o prácticas de clase en otras universidades para que participaran en el proyecto.

La fase 3 inició retomando los hallazgos que habíamos obtenido sobre la situación familiar así como sobre las preocupaciones escolares, familiares y de educación sexual de las adolescentes. Buscamos darle seguimiento a los avances en competencias verbales (oral y escrita) que se habían ejercitado con el proyecto de las fases 1 y 2 así como integrar nuevas variables de intervención a sugerencia tanto de las directivas del CSEVB como de las madres de familia. De este modo, se estableció como objetivo general comprender la problemática de los riesgos de deserción que se presentaban en el grupo de estudio y tratar de disminuirlos a través del empoderamiento de las adolescentes y de sus madres

TABLA 1.

Fases de IAP en el PAE del CSEVB

\begin{tabular}{|c|c|c|}
\hline FASE & INTERVENCION & INVESTIGACION \\
\hline $\begin{array}{l}\text { 1: Sensibilización } \\
\text { y capacitación } \\
\text { del equipo de } \\
\text { IAP (2009- } \\
\text { 2013). Esta fase } \\
\text { cs permanente, } \\
\text { se da a través } \\
\text { de reuniones } \\
\text { de trabajo e } \\
\text { intercambio, y } \\
\text { constituyc la } \\
\text { inducción para } \\
\text { los estudiantes } \\
\text { que se van } \\
\text { integrando cada } \\
\text { año al equipo de } \\
\text { lAP? }\end{array}$ & $\begin{array}{l}\text { Capacitación e inmersión } \\
\text { en cl cscenario de cstudio } \\
\text { por parte de los estudiantes } \\
\text { de la FCC involucrados cn } \\
\text { el proyecto, basado en los } \\
\text { objetivos de aprendizajc } \\
\text { y las comperencias } \\
\text { buscadas en el futuro } \\
\text { comunicólogo: investigación } \\
\text { cualitariva, intervención } \\
\text { para el desarrollo social, } \\
\text { vinculación (otras } \\
\text { universidades, la A.C.). } \\
\text { Durante el año 2010 } \\
\text { estuvo integrado a la } \\
\text { materia "Comunicación y } \\
\text { Educación" (Plan 265 de } \\
\text { la FCC) y desde entonces } \\
\text { al Servicio Social y al } \\
\text { PROMF.P (2012-2013), } \\
\text { ha sido problema de } \\
\text { investigación de } 3 \text { tesis de } \\
\text { licenciatura (en proceso) } \\
\text { y fue admitido como } \\
\text { actividad para la obtención } \\
\text { de créditos co-curricularcs } \\
\text { de estudiantes de la FCC. }\end{array}$ & $\begin{array}{l}\text { Formulación de un marco } \\
\text { tcórico y de referencia } \\
\text { sobre la deserción escolar, } \\
\text { las problemáticas de } \\
\text { adolescentes en situación } \\
\text { de riesgo, las políicas de } \\
\text { atención a la juventud en } \\
\text { Coahuila y la operación } \\
\text { de las OSC cn Saltillo. } \\
\text { Investigación documental } \\
\text { y visitas exploratorias al } \\
\text { escenario de estudio. }\end{array}$ \\
\hline $\begin{array}{l}\text { 2: Aprendizaje } \\
\text { colaborativo } \\
\text { (agosto } \\
2009 \text {-febrero } \\
2010 \text { ) }\end{array}$ & $\begin{array}{l}\text { Se impartió el taller } \\
\text { "historias de viday y } \\
\text { proyectos creativos", para } \\
\text { estudiantes de secundaria } \\
\text { y preparatoria. Resultado: } \\
\text { "Frase una vez", escritura } \\
\text { dc cuentos con temática de } \\
\text { salud (VIH). }\end{array}$ & $\begin{array}{l}\text { Inmersión y comprensión } \\
\text { socio-cultural del grupo } \\
\text { y de sus problemáticas, } \\
\text { búsqueda de problema de } \\
\text { investigación. } \\
\text { Técnicas aplicadas: } \\
\text { observación participante, } \\
\text { entrevistas informales, } \\
\text { bitácoras de rrabajo, } \\
\text { documentación. }\end{array}$ \\
\hline
\end{tabular}




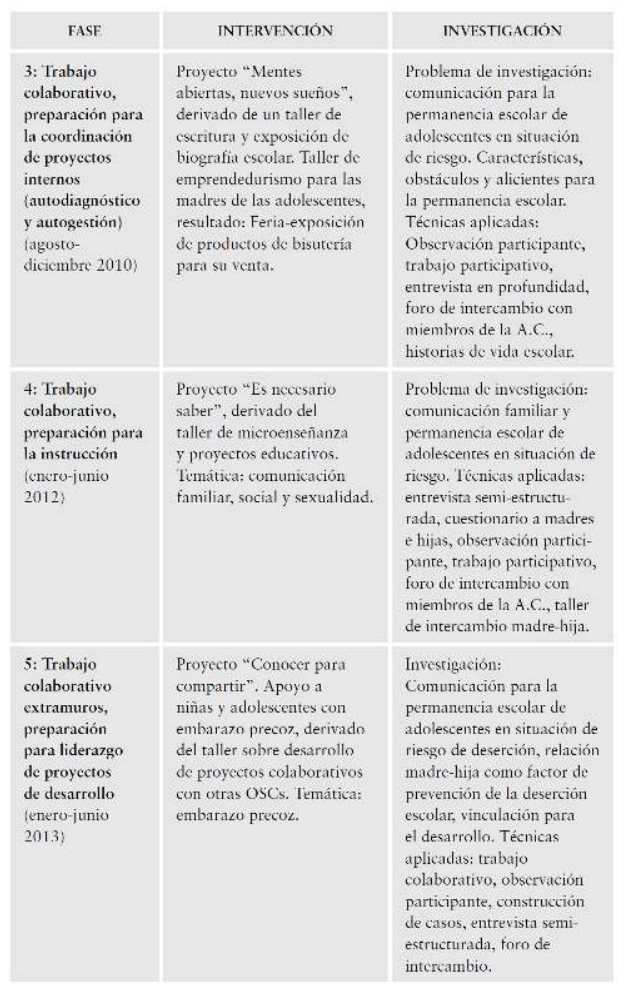

de familia, relación (madre-hija) que emergió como determinante en la permanencia escolar. Seguimos trabajando con el esquema de talleres, pero esta vez con la intención de que fueran ellas quienes decidieran y organizaran, una vez hecho el autodiagnóstico de su recorrido escolar, la forma y contenido de lo que compartirían de su experiencia con los demás miembros de la OSC. El taller para ellas giró en torno a dos ejes: autodiagnóstico escolar y organización de eventos públicos. También, se integró un nuevo equipo de estudiantes de la FCC que ofrecieron un curso de emprendedurismo a las madres de las adolescentes, cuyos resultados fueron expuestos, en formato de feria, el día del cierre del proyecto en el CSEVB. Por su parte, el foro de discusión -abierto después de la presentación de las biografías escolares de las participantes del PAE- resultó nuevamente un acontecimiento clave para su empoderamiento al recibir éstas retroalimentación positiva y sugerencias de mejoramiento por parte de todos los asistentes: madres y padres de familia, directivas del CSEVB, el equipo de IAP, prestadores de servicio social universitario y exbeneficiarias del PAE. A discusión abierta, fue posible la construcción de nuevas estrategias y acuerdos para la permanencia y la superación académica de las adolescentes. Así mismo, durante esta fase obtuvimos una caracterización clara de la problemática experimentada por las adolescentes en cuanto a su vida escolar, familiar y social con los instrumentos aplicados: un mapa de su historia de vida, entrevistas en profundidad aplicadas a las adolescentes, entrevista semi-estructurada aplicadas a las directivas de la OSC y observación participante. El título del evento y proyecto de esta fase fue elegida por ellas a través de una lluvia de ideas y resulta sugerente con respecto a su estado de ánimo sobre la permanencia escolar: "Mentes abiertas, nuevos sueños".

La fase 4 tuvo por objetivo reforzar sus competencias para el trabajo colaborativo así como prepararlas para su ejercicio como agentes de cambio con las beneficiarias del PAE que cursaban la primaria. El aprendizaje por modelamiento o aprendizaje social (Bandura, 1982) emergió como la teoría más pertinente para comprender y direccionar los procesos de cambio que se habían gestado a partir del proyecto de IAP (las adolescentes deseaban 
continuar estudiando como los alumnos de la FCC que iban cada sábado a impartir el taller, y las niñas pequeñas querían participar junto a las adolescentes en las actividades de estas últimas). El aprendizaje por modelamiento también parecía explicar la importancia de la relación madre-hija en la permanencia escolar al haber obtenido como resultado del cuestionario aplicado a ambas al finalizar la fase anterior, la introyección por parte de las adolescentes de la madre como modelo de superación y las madres asumían un rol como ejemplo y soporte para sus hijas. Por su parte, el equipo de IAP había venido trabajando desde el año 2009 bajo las teorías del aprendizaje constructivista, teniendo como fundamento las aportaciones de Vigotsky (cit. En Carretero, 2009), Savater $(1997,2000)$ y Paulo Freire (1973).

Durante esta etapa se sumaron por primera vez dos prestadoras de servicio social de la FCC y una tesista que eligió como tema de investigación el impacto de la comunicación efectiva en la permanencia escolar de las mujeres adolescentes en situación de riesgo de deserción ${ }^{11}$. El taller que se impartió en esta etapa fue sobre microenseñanza, por un lado, y de comunicación asertiva, por otro. Se contó con nuevos instructores además de los estudiantes de la FCC: prestadores de servicio social de la preparatoria del ITESM y 2 especialistas en el tema de "valores y relaciones sociales". De forma participativa, se decidió nuevamente compartir con los miembros del CSEVB la experiencia de los talleres a través de un evento público, así como diseñar actividades para fomentar los valores y la educación en familia, la comunicación asertiva y el desarrollo humano. El evento fue diseñado por el equipo de IAP (conformado por los actores sociales del PAE, de la FCC y del CSEVB) con base en dos temas que las adolescentes priorizaron para su propio desarrollo: educación sexual y comunicación familiar. Mientras diseñábamos los objetivos del evento, una de las adolescentes derivó de ahí el título del mismo, que fue aprobado por unanimidad por el grupo: "Es necesario saber". La presentación final inició con un ejercicio guiado entre cada pareja de madre e hija, donde de forma confidencial, se les pidió que conversaran sobre las siguientes preguntas que les fueron entregadas:

a. A la hija: ¿Qué me gustaría que me explicara o me contara mi mamá sobre las relaciones amorosas y sobre mi desarrollo en la sexualidad?

41 b. A la mamá: ¿qué desea para su hija en un futuro próximo?, ¿qué desea para su hija en cuanto a una relación amorosa?, ¿qué cosas de una relación amorosa considera que su hija necesita saber de su boca?

Posteriormente, se procedió a tener dos conferencias con los especialistas en desarrollo humano, que incluyeron actividades de comunicación asertiva en la familia y algunas preguntas para la reflexión. El cierre consistió en la entrega de una carta que previamente habían escrito las adolescentes para sus madres (estaba cercana la fecha del Día de la Madre) y en un foro abierto de discusión sobre lo dialogado durante el evento. Incluimos, al finalizar, el llenado de un cuestionario con el fin de complementar la investigación sobre la comunicación familiar y de obtener algunos datos sobre el impacto de la intervención. Por último, tuvimos una junta de retroalimentación con las adolescentes para decidir el rumbo del trabajo en la siguiente etapa.

Fase 5. Durante los meses en que transcurrió esta etapa, decidimos que actuaríamos con base en un modelo de mejora continua, cuya derivación natural sería tener por objetivo que las adolescentes lograran la autogestión para el diseño de un proyecto de desarrollo que, al mismo tiempo en que les permitiera profundizar en los temas que ellas detectaban como importantes para su desarrollo, pudieran llevar sus hallazgos y su participación a otros contextos donde se presentara la misma problemática. De este modo, inició la 
preparación para su intervención extramuros. El taller consistió en la investigación, presentación oral así como discusión grupal sobre algunos modelos de participación propios de los OSC. El equipo de IAP presentó la fundamentación teórico-práctica del Tercer Sector en México así como algunos ejemplos de acción comunitaria por parte de OSCs internacionales, nacionales y regionales. Las adolescentes tendrían que diseñar, junto al equipo de IAP, un proyecto de intervención que beneficiara a otras OSCs y que les permitiera aproximarse a un trabajo cooperativo extramuros, aprovechando que ya tenían prácticas previas en la organización de proyectos para el CSEVB. En esta etapa se integraron al equipo dos voluntarias más que habían diseñado, desde una agrupación universitaria del ITESM, un proyecto de acción social en apoyo a una OSC saltillense que, junto con el DIF, daba atención a niñas y adolescentes con embarazo precoz. Su proyecto no había podido llevarse a cabo en dicha A.C debido a problemas de inseguridad pública (el local-albergue había sido objeto de robo y no se contaba con medidas de seguridad que pudieran garantizar la integridad de sus beneficiarias) y buscaban implementarlo en algún otro contexto que enfrentara una problemática similar. Al mismo tiempo, el embarazo precoz seguía apareciendo como preocupación constante de las adolescentes del PAE y como principal factor de riesgo de deserción femenina tanto en la literatura como en la historia del mismo PAE. Fue durante este semestre que el proyecto fue aprobado por el PROMEP y recibió recursos adicionales para su realización, se sumaron 3 prestadoras más de servicio social de la FCC así como se integraron 2 tesistas cuyos temas de investigación versaban sobre el impacto de la comunicación familiar en la permanencia escolar de adolescentes en situación de riesgo así como en el diseño de una campaña social a favor de la permanencia escolar de estudiantes de secundaria y preparatoria en Saltillo ${ }^{12}$.

El grupo de IAP decidió tener como proyecto de desarrollo en esta fase, el diseño de una campaña de información-concientización sobre la problemática del embarazo precoz en apoyo a las niñas y adolescentes de la otra OSC saltillense con la que habíamos entrado en contacto. El esquema de IAP incluyó para cada sesión sabatina el avance de conocimientos para la construcción de una propuesta de intervención cooperativa así como la recopilación de datos para la investigación sobre el papel de la comunicación familiar en la permanencia escolar. Los temas presentados y discutidos fueron los que se mencionan a continuación y siguieron la lógica de un trabajo que va desde el fortalecimiento personal y comunitario de las adolescentes como agentes sociales hasta los saberes prácticos para la implementación de un proyecto de comunicación para el desarrollo: autoestima y cuidado de la salud (impartido por una especialista externa), trabajo en equipo (impartido por las desarrolladoras del ITESM) y gestión de proyectos sociales a través de redes sociales (impartido por una egresada de la FCC). Bajo un esquema de información-discusión-práctica, las adolescentes desarrollaron una Fanpage interactiva e informativa para Facebook sobre el tema de embarazo precoz. Aunque también estaban proyectadas actividades de intervención para la campaña, no fue posible realizarlas en esta fase, pues la OSC beneficiaria fue cancelada y no pudimos concretar los permisos para hacer trabajo de campo en la Ciudad con fines de difusión. No obstante, se compartió de nuevo el proyecto con los miembros del CSEVB a través de un evento público que incluyó, como reforzamiento y evaluación del área de comunicación familiar, una dinámica de diálogo e intercambio entre las adolescentes y sus madres. Se repartieron al azar temas para ser discutidos en dicha pareja, y se les pidió a las niñas que, utilizando sus conocimientos de comunicación asertiva, comunicaran a sus madres la noticia escrita en el papel ${ }^{13}$ y que compartieran con ellas los contenidos que habían discutido en el taller 
con los especialistas. El título de este proyecto semestral fue "Conocer para compartir", que expresaba el camino recorrido en su aproximación al liderazgo de proyectos de desarrollo social a través de la comunicación.

Posterior al cierre de intervención de la fase 5, fue posible resumir y analizar la experiencia acumulada hasta entonces a través de la elaboración de una ponencia que fue presentada en el V Foro de Investigación de la Facultad de Trabajo Social de la UAdeC, donde participamos 4 miembros del equipo de IAP ${ }^{14}$.

La siguiente etapa está por comenzar, siguiendo el modelo presentado al inicio de este subtema y que habrá de retomar las conclusiones de la fase 5.

\section{Conclusiones}

El modelo de IAP que presentamos en este trabajo ha resultado lo suficientemente flexible para poder ser retomado en las distintas fases de fortalecimiento comunitario y profundización de las categorías que han emergido durante la investigación. Consideramos que, si se sostienen los siguientes fundamentos, puede ser replicado para otros proyectos de IAP con otros grupos socioculturales:

a. La investigación-acción no puede realizarse sin una permanente práctica ética.

b. La investigación-acción debe ser participativa, participante y justa.

c. Las técnicas cualitativas, a partir de una estrategia de abordaje humanista, representan una herramienta de enorme valor para profundizar en las problemáticas de los grupos socioculturales desde una aproximación microsocial.

d. La investigación-acción requiere de competencias adicionales a las del manejo de las técnicas de intervención o investigación, tales como: vinculación, negociación, seguimiento.

Sobre la relación familia-permanencia escolar de adolescentes en situación de riesgo de deserción, podemos adelantar algunos hallazgos con los que ya contamos. Los factores que apoyan la permanencia escolar en nuestro grupo de estudio, son los siguientes y se combinan como parte de una problemática compleja que incluye lo económico, afectivo, académico y social. Las adolescentes en situación de riesgo de deserción tienen mayor probabilidad de permanecer en el sistema educativo formal, si:

a. Existe apoyo de tutores: comunicación dialógica y relaciones de afecto con padres o fi guras de autoridad en la unidad doméstica.

b. La adolescente forma parte de una familia que se coloca culturalmente en pro de la educación.

c. Existe una estrecha relación madre-hija.

d. Existe un discurso y una práctica de estabilidad familiar, entendidas como la sana interacción (comunicación asertiva) entre miembros de la familia, donde los padres o tutores mantienen un discurso de confianza económica basada en el desarrollo de sus miembros y de esperanza con respecto a la formación escolar de sus hijos.

57 Resulta pertinente, así mismo, retomar las estadísticas de estancia y abandono de las adolescentes beneficiarias del PAE: de 2010 a la fecha, el $60 \%$ de ellas ha abandonado el programa. De un grupo permanente de 10, 2 abandonaron por falta de recursos económicos -la tutora, abuela de las adolescentes, no pudo seguir costeando su educación 
secundaria y preparatoria-, 1 abandonó el programa por falta de apoyo de tutores falleció su abuelita, que era quien se hacía cargo de su manutención y no encontró soporte en otros miembros de su familia-, 1 más terminó sus estudios de preparatoria y su padre no la autorizó a seguir estudiando; se casó, trabaja y es madre de familia, otra presentó embarazo precoz y abandonó sus estudios de secundaria y, finalmente, otra de ellas dejó de asistir por baja escolar. Estos espacios son cubiertos por nuevas beneficiarias del PAE, de modo que tanto las niñas del programa que pasan de primaria a secundaria se integran a este grupo de adolescentes, como son revisadas nuevas propuestas de estudiantes para entrar al CSEVB. Por otro lado, los casos que se consideran de éxito son los de aquellas adolescentes que han logrado una permanencia en el PAE por más de 6 años (70\% de ellas), donde además, en el 2013 dos de ellas lograron ingresar al nivel universitario.

El seguimiento que se tiene planeado para este modelo de IAP incluye la continuación de proyectos de desarrollo con el grupo de estudio (consolidar su autogestión), así como con otras OSC de Saltillo que trabajan con adolescentes en situación de riesgo de deserción. En cuanto a la investigación sobre el papel de la comunicación en la permanencia y superación escolar, resulta conveniente extender el análisis a otros grupos así como ahondar en el fenómeno desde la teoría del aprendizaje social, significativo y constructivista. Así mismo, ante el actual panorama escolar y social, no se puede soslayar el impacto que las brechas digitales (Alva de la Selva, 2012) podrían estar teniendo sobre los índices de deserción escolar adolescente, aproximación de la cual se proyecta derivar algunas variables que se relacionen de forma directa con el marco de acción de nuestra comunidad de estudio.

\section{BIBLIOGRAFÍA}

ALCOCER, M. (1998), “Investigación acción participativa”, en Galindo, J., Técnicas de investigación en sociedad, cultura y comunicación. México, DF: Pearson Addison Wesley.

ALVA DE LA SELVA, A. R. (2012), Brecha e inclusión social en México: hacia una propuesta de políticas públicas. México, DF: UNAM.

ÁLVAREZ-GAYOU, Jurgenson, J. L. (2010), Cómo hacer investigación cualitativa. Fundamentos y metodología. México, DF: Paidós Educador.

BANDURA, A. (1982),Teoría del aprendizaje social. Madrid: Espasa-Calpe.

BELTRÁN, L. (2005), “La Comunicación para el Desarrollo en Latinoamérica: Un recuento de medio siglo", en III Congreso Panamericano de la Comunicación. Panel 3: Problemática de la Comunicación para el Desarrollo en el contexto de la Sociedad de la Información (pp. 9-16). Buenos Aires: Universidad de Buenos Aires.

BLUMER, H. (1969), Symbolic Interactionism: Perspective and Method. Berkeley: University of California Press.

CARRETERO, M. (2009), Constructivismo y educación. Buenos Aires: Paidós. 
CORDERO, D. (1999), "La salud como factor fundamental para la equidad e igualdad de la mujer. Alternativas comunicacionales comunitarias" en Cicalese, G. (1999). Comunicación comunitaria. Apuntes para abordar las dimensiones de la construcción colectiva. Buenos Aires: La Crujía.

ENOE-INEGI (2010), Encuesta Nacional de Ocupación y Empleo. Recuperado de http:// www.inegi.org.mx/est/contenidos/Proyectos/encuestas/hogares/regulares/enoe/, el 22 de diciembre de 2013.

FREIRE, P. (2009), La educación como práctica de la libertad. México, DF: Ed. Siglo XXI.

FREIRE, P. (2007), Pedagogía de la esperanza. México DF: Ed. Siglo XXI.

FREIRE, P. (2006), Pedagogía del oprimido. México, DF: Ed. Siglo XXI.

FREIRE, P. (1973), ¿Extensión o comunicación? La concientización en el medio rural. México, DF: Editorial Siglo XXI.

HERNÁNDEZ SAMPIERI, R.; FERNÁNDEZ-COLLADO, C. y BAPTISTA, P. (2010), Metodología de la investigación, $5^{\text {a }}$. edición. México: Mc. Graw Hill.

IMJUVE (2005), Encuesta Nacional de la Juventud. Recuperado de http:// cendoc.imjuventud.gob.mx/investigacion/encuesta.html el 22 de enero de 2013.

INEGI (2013), Censo General de Población y Vivienda del 2000. Recuperado de http:// www.inegi.org.mx/est/contenidos/Proyectos/ccpv/cpv2000/default.aspx el 6 de enero de 2013. INEGI (2013), Estadísticas de Natalidad. Recuperado de http://www3.inegi.org.mx/sistemas/sisept/ default.aspx?t=mdemo29\&s=est\&c=21882 el 19 de diciembre de 2013.

INEGI (2011), Resultados del II Censo de Población y Vivienda. Recuperado de http:// www.inegi.gob.mx/prod_serv/contenidos/espanol/bvinegi/productos/censos/poblacion/2010/ princi_result/cpv2010_principales_resultadosII.pdf el 30 de octubre de 2012.

KAPLÚN, J. (1987), El comunicador popular. Culturas juveniles y educación: pedagogía crítica, estudios culturales e investigación participativa, en Los jóvenes: múltiples miradas, UNC. 10pp.

KAPLÚN, M. (1997), “La educomunicación. De medio y fi nes en comunicación”. Revista Latinoamericana de Comunicación Chasqui. Número 58. Recuperado el 8 de agosto de 2010 de http:// chasqui.comunica.org/kaplun.htm

MURCIA PEÑA, N., JARAMILLO ECHEVERRY, L. G. (2000), Investigación cualitativa. Una guía para abordar estudios sociales. Armenia: Editorial Kinésis.

NAVARRO, N. L. (2001), Marginación escolar en los jóvenes. Aproximación a las causas de abandono. Recuperado el 15 de febrero de 2012, de http://www.inegi.gob.mx/inegi/contenidos/espanol/ prensa/contenidos/articulos/sociodemografi cas/marginacion.pdf.

SALINAS, B. (1994), "Educación popular, comunitaria e investigación participativa", en Los estados del conocimiento. México. DF: IPN, Departamento de Investigación Educativa del CINVESTAV.

SAVATER, Fernando (1997), El valor de educar. México, DF: Instituto de Estudios Educativos y Sindicales de América.

----- (2000), Los caminos para la libertad. Ética y educación. México, DF: Cátedra Alfonso Reyes del Tecnológico de Monterrey y Editorial Planeta Mexicana, S.A. de C.V.

SEP y COPEEMS (2012), Reporte de la Encuesta Nacional de Deserción de Educación Media Superior Recuperado de http://www.siguele.sems.gob.mx/encuesta.php el 22 de diciembre de 2013. 
SEP (2012), Sistema Educativo de los Estados Unidos Mexicanos. Principales cifras, ciclo escolar 2011/2012. Obtenido el 19 de diciembre de 2013 de http://www3.inegi.org.mx/sistemas/sisept/default.aspx? $\mathrm{t}=$ medu24\&s=est\&c=21784.

STRAUSS, A. y CORBIN, J. (2002), Bases de la investigación cualitativa. Técnicas y procedimientos para desarrollar la teoría fundamentada. Antioquia: Universidad de Antioquia.

\section{NOTAS}

1. Por arriba del promedio nacional, que alcanza un $19,4 \%$ de nacimientos de hijos de madres adolescentes en el año 2012, de acuerdo con las estadísticas de natalidad del INEGI (2013).

2. Conclusiones provisionales sobre los factores culturales que inciden en la deserción escolar de mujeres adolescentes en Saltillo, extraídas del trabajo de campo que se realiza para este proyecto de investigación-acción del año 2010 a la fecha. Dentro de los proyectos de promoción social desarrollados desde una perspectiva de género, la discriminación escolar femenina derivada de una cultura patriarcal es considerada como variable de primer orden para ser intervenida (Cordero, 1999).

3. Fundación Oportunidades Educativas, A.C; Programa Apoyo a la Esperanza del Centro de "Superación Estela V. Barragán", A.C; y La Casa de los Niños, A.C como las tres iniciativas de la sociedad civil más sólidas en la prevención de la deserción escolar en Saltillo, Coah.

4. Hablamos, particularmente, de una metodología basada en los fundamentos de la investigación-acción participativa como la descrita por Alcocer (1998) y desarrollada como filosofía pedagógica por Freire (1973, 2006, 2007, 2009).

5. Alcocer (1998) refiere al trabajo de Bertha Salinas (1994) como una síntesis de las experiencias de proyectos de investigación-acción en nuestro país.

6. Se eligió al CSEVB a partir de los objetivos con que éste cuenta: ser un espacio de desarrollo académico para las mujeres de Saltillo, sin distinción de edad, preferencia política, religión o formación educativa.

7. La Profra. Estela V. Barragán de la Fuente (Saltillo, 1917-2005) se tituló como Profesora Normalista de Primaria, posteriormente cursó las maestrías de Lengua y Literaturas Españolas y de Pedagogía Técnica en Educación en la Escuela Normal Superior de Coahuila. Terminó la carrera de Psicología Educativa, rama Orientador, en la Normal Superior de la Ciudad de México y realizó estudios de Posgrado en la Universidad de Oklahoma y en el Instituto Politécnico de Virginia, EEUU. Primero ejerció como profesora de primaria y después atendió cátedras en el Bachillerato del Ateneo Fuente, en la Preparatoria Nocturna y en la Normal Superior de Saltillo. Introdujo y difundió la Orientación Vocacional en el Estado de Coahuila y en otros estados aledaños. Fue Dirigente Estatal Femenil del PRI de 1964 a 1968 y Diputada Local de 1963 a 1966. Fue coautora de diverso folletos educativos sobre Paternidad Responsable e Integración Familiar así como autora de los libros "Conociendo a Coahuila", "Una familia de Saltillo" y "Guía de oportunidades educativas en Coahuila". Recibió la "Presea Saltillo" en 1996, la "Presea IMARC" en 1998 y en Julio de 2001 el Gobierno del Estado le otorgó un reconocimiento por su labor humanitaria y educativa. En diciembre del mismo año se realizó la inauguración del Recinto del CSEVB en un terrero donado por el Gobierno Estatal y le fue asignado en custodia el terreno donde en el 2002 se inauguró el "Jardín de las Mujeres", que albergaba los bustos en bronce de 5 mujeres saltillenses que habían destacado en distintos ámbitos de la comunidad, incluida la Profra. Estela V. Barragán.

8. Actualmente el proyecto se encuentra en etapa de análisis y presentación de resultados sobre los factores socio-culturales y económicos que inciden en la permanencia escolar del grupo de estudio-intervención, así como del papel que juegan las habilidades de comunicación 
interpersonal de las adolescentes en sus ámbitos familiar, educativo y extraescolar para la superación académica. El análisis no ha sido publicado, pero puede ser solicitado a la autora.

9. No solamente por el intercambio de conocimientos que se produce entre el equipo investigador (con herramientas de indagación y análisis de información) y la comunidad de intervención-estudio (con el conocimiento que tienen de sí mismos y de los problemas que enfrentan), sino porque la participación comprometida de ambos genera procesos de aprendizaje que enriquecen tanto el abordaje de los problemas como la creación de metodologías flexibles para su solución a mediano y largo plazo. Este involucramiento con perspectiva científica y humanista, además, conduce a sus participantes a efectos multiplicadores de desarrollo social así como permite al investigador profundizar en y ramificar temas desde una línea de investigación anclada en un fragmento de realidad social que admite una experimentación que está llamada a ser ética, pertinente y permanente.

10. El término "espiral" aparece repetidamente en la literatura sobre la investigación-acción. Fue acuñado por el psicólogo germano-norteamericano Kurt Lewin en la década de los cuarenta para referirse a la mutua influencia de los miembros de grupos pequeños en la toma de decisiones. Con el término "espiral" nos referimos en este trabajo a la posibilidad de que conforme avanza el proceso participativo de concientización-acción, es posible ir profundizando en los orígenes y las consecuencias de la problemática de estudio, así como ir integrando a agentes sociales de otros sectores y campos de especialidad científica y/o de saber popular.

11. Puede revisarse el trabajo de Silvia Nohemí de la Cruz Ruiz, "Comunicación interpersonal y su impacto en el diseño y ejecución de proyectos educativos para adolescentes en situación de riesgo en Saltillo" que fue presentado en II Encuentro Nacional sobre Comunicación y Juventud "Jóvenes y participación social" de la Facultad de Ciencias Antropológicas de la Universidad Autónoma de Yucatán (Mérida, Yucatán; 9 de noviembre de 2012).

12. Puede solicitarse información a las autoras de dichas tesis: Victoria Elizabeth Ibarra Castillo y Stefany Nájera Estrello.

13. Los temas fueron distribuidos al azar en ese momento, e incluyeron situaciones en los temas de desarrollo sexual, afectivo, escolar, familiar y social.

14. Gabriela de la Peña Astorga, Silva Nohemí de la Cruz Ruiz, Ana Luisa Hernández Casas y Gibrán Alejandro Valdez Flores (2013). "Comunicación para la permanencia escolar de mujeres adolescentes en situación de riesgo de deserción en Saltillo: investigación-acción en el Centro de Superación Estela V. Barragán, A.C." V Foro de Investigación en Trabajo Social. Facultad de Trabajo Social de la Universidad Autónoma de Coahuila. Saltillo, Coah; 18 de abril de 2013.

\section{RESÚMENES}

Este artículo muestra la fundamentación teórica, metodológica y empírica que guio la elaboración de un modelo de investigación-acción participativa y su aplicación al área de comunicación para la permanencia escolar de mujeres adolescentes en situación de riesgo de deserción en Saltillo en el marco del "Programa Ayuda a la Esperanza" del Centro de Superación “Estela V. Barragán”, A.C.

Este artigo mostra a fundamentação teórica, metodológica e empírica que guiou a elaboração de um modelo de investigação-ação participativa e sua aplicação na área da comunicação para a 
retenção escolar de mulheres adolescentes em situação de risco de abandono escolar em Saltillo, no quadro do "Programa Ajuda à Esperança" do Centro de Superación Estela V. Barragán, A.C.

This paper approaches the theoretical, methodological and empirical groundings that guided the construction of a model of Participatory Action Research as well as its application to the communication field for school retention of adolescent girls at risk of dropping out in Saltillo in the frame of the Help for Hope Program of the Center for Academic Development "Estela V. Barragán", A.C.

\section{ÍNDICE}

Keywords: Participatory Research Action, teen dropouts, communication studies, social development, Saltillo

Palabras claves: Investigación-acción, deserción escolar, mujeres adolescentes, comunicación para el desarrollo

Palavras-chave: Investigação-ação, abandono escolar, mulheres adolescentes, comunicação para o desenvolvimento

\section{AUTOR}

\section{GABRIELA DE LA PEÑA ASTORGA}

Facultad de Ciencias de la Comunicación de la Universidad Autónoma de Coahuila, México.

Enderezo de correspondencia: Miravalle \#439. Col. Jardines de Valle Saltillo, Coah. México C.P. 25260

gabriela.pena@uadec.edu.mx 